(C) 2017 by the Arizona Board of Regents on behalf of the University of Arizona. This is an Open Access article, distributed under the terms of the Creative Commons Attribution licence (http://creativecommons. org/licenses/by/4.0/), which permits unrestricted re-use, distribution, and reproduction in any medium, provided the original work is properly cited.

\title{
CULTURE PROCESS AND THE INTERPRETATION OF RADIOCARBON DATA
}

\author{
Jacob Freeman $^{1 *} \cdot$ David A Byers $^{1} \cdot$ Erick Robinson $^{2} \cdot$ Robert L Kelly $^{2}$ \\ ${ }^{1}$ Anthropology Program, Utah State University, 0730 Old Main Hill, Logan, UT 84321 USA. \\ ${ }^{2}$ Department of Anthropology, University of Wyoming, 12th and Lewis Street, Dept. 3431, 1000 E. University \\ Avenue, Laramie, WY 82071 USA.
}

\begin{abstract}
Over the last decade, archaeologists have turned to large radiocarbon $\left({ }^{14} \mathrm{C}\right)$ data sets to infer prehistoric population size and change. An outstanding question concerns just how direct of an estimate ${ }^{14} \mathrm{C}$ dates are for human populations. In this paper we propose that ${ }^{14} \mathrm{C}$ dates are a better estimate of energy consumption, rather than an unmediated, proportional estimate of population size. We use a parametric model to describe the relationship between population size, economic complexity and energy consumption in human societies, and then parametrize the model using data from modern contexts. Our results suggest that energy consumption scales sub-linearly with population size, which means that the analysis of a large ${ }^{14} \mathrm{C}$ time-series has the potential to misestimate rates of population change and absolute population size. Energy consumption is also an exponential function of economic complexity. Thus, the ${ }^{14} \mathrm{C}$ record could change semi-independent of population as complexity grows or declines. Scaling models are an important tool for stimulating future research to tease apart the different effects of population and social complexity on energy consumption, and explain variation in the forms of ${ }^{14} \mathrm{C}$ date time-series in different regions.
\end{abstract}

KEYWORDS: deep-time economics, macroecology, prehistoric demography, summed probability distribution.

\section{INTRODUCTION}

The objective of this paper is to critically discuss how to learn about prehistoric social and demographic processes from large samples of radiocarbon $\left({ }^{14} \mathrm{C}\right)$ dates. Archaeologists increasingly use large samples of ${ }^{14} \mathrm{C}$ dates to estimate human population sizes, long-term population growth rates, and other demographic processes (e.g., Pettitt et al. 2003; Shennan 2008; Peros et al. 2010; Williams 2012, 2013; Kelly et al. 2013; Shennan et al. 2013; Contreras and Meadows 2014; Wang et al. 2014; Crema et al. 2016; Zahid et al. 2016). Making inferences from these data sets about demography, however, is not without challenges (Williams 2012; Downey et al. 2014; Attenbrow and Hiscock 2015; Brown 2015, 2017). The issues stem from processes external and internal to prehistoric human populations. The majority of archaeological studies focus on external processes that may bias inferences about population from ${ }^{14} \mathrm{C}$ date time-series, such as the effects of site sampling, sample size, ${ }^{14} \mathrm{C}$ date calibration and preservation bias (e.g., Surovell et al. 2009; Williams 2012; Contreras and Meadows 2014; Shennan 2013; Brown 2015). These studies constitute invaluable frames of reference for making more informed inferences about demographic processes from the frequency of ${ }^{14} \mathrm{C}$ date time-series. However, less attention has been paid to the ways that cultural process, internal to prehistoric populations, may affect the creation of the ${ }^{14} \mathrm{C}$ record (but see Downey et al. 2014).

Simply put, fundamental changes in the basic contours of a society might prompt shifts in the relationship between population size and the datable materials that people produce. As a first step in exploring this issue, we model the effects of population size and economic complexity on the production of waste products that archaeologists date, and explore how it might confound the ways we currently make inferences about demographic and other cultural dynamics from the frequency distribution of large samples of ${ }^{14} \mathrm{C}$ dates. We use modern data to parametrize the model and, from our results, make two points relevant to the study of dates as data. (1) ${ }^{14} \mathrm{C}$ date

\footnotetext{
*Corresponding author. Email: jacob.freeman@usu.edu.
} 
frequencies arrayed in a time-series, based on large samples of dates, probably misestimate rates of population growth. This is because ${ }^{14} \mathrm{C}$ dates, all else equal, are one measure of the energy consumed by prehistoric populations, and the scaling relationship between population and energy consumption is often sub-linear in human populations. (2) Understanding the previous point permits researchers to make predictions about how the ${ }^{14} \mathrm{C}$ record covaries with other classes of material culture and further evaluate the importance of changes in energy consumption in prehistoric social change.

\section{Dates as Data and Demography}

The premise behind using the frequency of ${ }^{14} \mathrm{C}$ dates in a time-series to infer population trends is that the number of person years a region is occupied is proportional to the production of cultural waste that archaeologists date. As Rick (1987:54) states,

Despite intervening biases, I assume that the number of dates is related to the magnitude of occupation, or to the total number of person-years of human existence in a given area. Using this premise, it is possible to assess and compare, in a relative fashion, the occupation histories within and between regions.

This is a reasonable starting point, but there is a basic unevaluated assumption. ${ }^{1}$ Following Rick's initial assumption, most studies assume that population size and the frequency of ${ }^{14} \mathrm{C}$ dates produced by prehistoric populations are proportional (Peros et al. 2010:659). A proportional relationship simply means that the production of ${ }^{14} \mathrm{C}$ by a population is a constant multiple of population size. A subtle ancillary assumption of this relationship between population size and waste production is that individuals in a population are autonomous, and their production and consumption decisions that result in waste lack mutual interdependence.

This may be a poor assumption. Social networks and technological differences can create efficiencies of scale that lead to a sub-linear relationship between population and the consumption of energy, materials and information in human societies (Hamilton et al. 2007; Freeman and Anderies 2015). And, the consumption of energy, whether burning wood to stay warm or consuming bone marrow to maintain metabolic needs, drives the accrual of datable materials. We simply do not know whether the accumulation of cultural debris, due to the consumption of energy, and population size are related in a proportional manner, and this may bias our ability to make inferences about demographic processes from large samples of ${ }^{14} \mathrm{C}$ dates. Thus, to make inferences from such data, we need to build models for understanding how population and energy consumption are related.

\section{An Energy Consumption Model}

To build a model that scales population and the production of datable materials we assume the following: all else equal, the frequency of ${ }^{14} \mathrm{C}$ dates collected via unbiased sampling is one estimate of the quantity of energy consumed by prehistoric populations, and the waste products that result are proportional to the total amount of energy consumed. These assumptions are simple, but, we argue, more reasonable than assuming ${ }^{14} \mathrm{C}$ date frequencies are an unmediated reflection of population. These assumptions simply rest on the premise that the materials we date (bone, charred wood and seeds, etc.) are the byproducts of energy consumption events that occur as populations live and reproduce, because biological maintenance and reproduction

\footnotetext{
${ }^{1}$ See Attenbrow and Hiscock (2015) for different critiques.
} 
require energy. The more of such events that take place during a given time period, the more likely it is that organic waste products will preserve and accumulate.

Given the above assumptions, we propose a model of changes in energy consumption that shares the same structure as a widely used macroeconomic model of human impacts on ecosystems (York et al. 2003). Formally,

$$
E=M P
$$

where $E$ is total energy consumed; $M$ describes the energy necessary for an average individual to live and reproduce; and $P$ is population.

We assume that $M$ has two components. The first is a constant metabolic rate defined by human biology that has evolved over the last two million years. The second comportment is economic complexity, which changes the metabolic rate that is necessary for an average individual to live and reproduce in a given social system. Complexity is a much debated concept in anthropology that can invoke many different ideas, such as scalar changes in political hierarchies to differences in economies. Here, we follow the definition of Hidalgo and Hausmann (2009:10,570) and define economic complexity as the number of capabilities and degree of integration among those capabilities in an arbitrarily bound economy. A capability is simply the ability to make something or provide a service. The degree to which capabilities are integrated refers to how much investment has been made to link different capabilities together via institutions and organizations. We adopt this view, in part, because the linkage between energy consumption and the accumulation of capabilities in a socioeconomic system is intuitive. However, it is only a starting point.

As the number of economic specialties and investments in organizations and institutions that integrate those specialties increases, populations should consume more energy. To take an extreme example, not only do modern post-industrial economies have steel workers, IT specialists, farmers and engineers, but populations in such an economy also invest in creating networks of organizations and institutions that link these specialists, such as corporations, government bureaucracies, schools and social clubs. All of these organizations require institutions to run effectively (which means energy invested in socialization) and built infrastructure, like structures, fiber-optic lines and computers, to function (which requires energy to build and maintain).

We assume here that biological metabolism is a constant across human populations (i.e., varies much less than complexity). Holding biological metabolism constant, the energy necessary per person should scale with complexity, $C$; where $C$ is the number of capabilities and level of integration of those capabilities in a system. In mathematical notation, we write the effect of $C$ on energy consumption as an increasing exponential function:

$$
M=m_{1} e^{\beta_{1} C}
$$

where $\mathrm{m}_{1}$ is the constant biologically determined metabolic rate (energy per person per unit time); and $\beta_{1}$ is a coefficient that scales the rate of change in energy consumption per unit increase in complexity, $C$. Here, $C$ is constrained to positive integers, and $\beta_{1}>0$. Equation 2 and its constraints mean that the consumption of energy compounds exponentially as the number of capabilities and the degree those capabilities are integrated in an economy increases (Figure 1a). Where the economy is very simple (has only one capability), then per capita energy consumption is very near an individual's biological metabolic rate. As $C$ increases, however, the consumption 
(a)

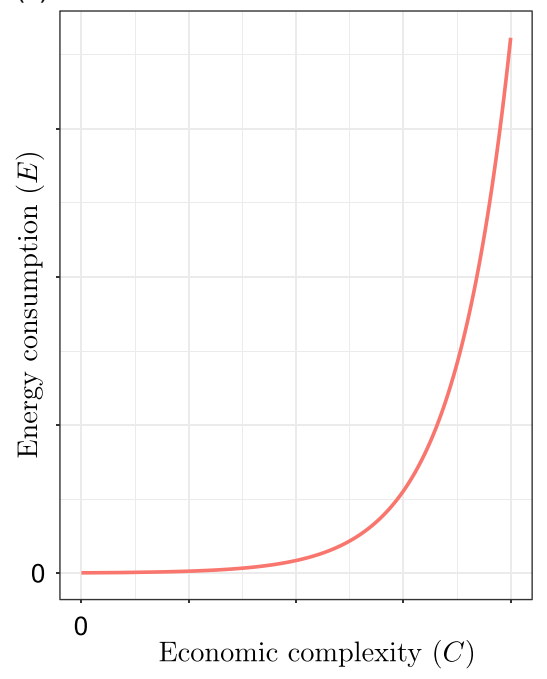

(b)

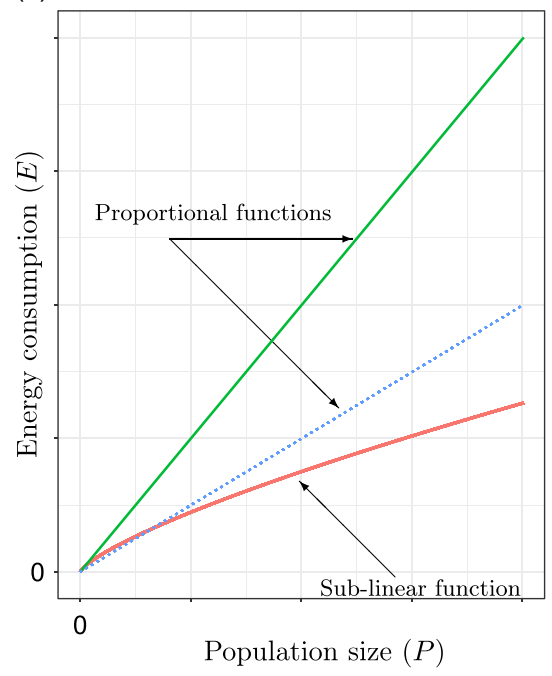

Figure 1 (a) The proposed relationship between economic complexity and total energy consumption. (Note that we use a continuous function simply to illustrate the shape of the relationship; $C$ is constrained to positive integers.) (b) Potential relationships between population and total energy consumption. The standard assumption in current research is that the relationship is proportional (green dashed line and blue dotted line). We propose that the relationship is sub-linear (red solid line); as population increases, the marginal increase in energy consumption decreases. (See online version for colors.)

of energy necessary for a given population to live and reproduce compounds exponentially to account for all of the new specialties in an economy and the integration of those specialties.

Holding complexity equal, Equation 1 captures the basic assumption that population scales linearly with total energy consumption, and that individuals in a population do not interact in ways that create increasing or decreasing efficiencies in the consumption of energy. Our working hypothesis here is that the relationship between population and energy consumption is sub-linear rather than in proportion as Equation 1 assumes. This means that adding one more individual to a foraging camp does not require a constant increase, but, rather, a decelerating increase in the amount of wood needed to keep that individual warm (see Figure 1b). We suspect that the relationship is sub-linear because of previous work on territory size in human societies.

Not unlike our assumption that the frequency of ${ }^{14} \mathrm{C}$ dates estimates energy consumption, ecologists have long assumed that the size of an animal's range (territory) is an estimate of the energy that an average individual needs to consume (McNab 1963; Milton and May 1976; Lindstedt et al. 1986; Brown et al. 2004; Jetz et al. 2004). For instance, the larger an animal's body size, the larger its range because big animals consume more energy than small animals (McNab 1963). Models of animal territory size applied to hunter-gatherers and subsistence agricultural societies also assume that the size of a group's territory is an emergent outcome of the area needed to consume energy by a population (Hamilton et al. 2009, 2007; Freeman and Anderies 2015; Freeman 2016). These studies indicate that, among both hunter-gatherers and agriculturalists, territory size is often a sub-linear function of population size (Figure 2). Why this is the case remains an open question, but current hypotheses concur that as population size increases, individuals become more efficient consumers of energy and information and, thus, have more overlapping individual home-ranges (Hamilton et al. 2009; Freeman and Anderies 2015). 


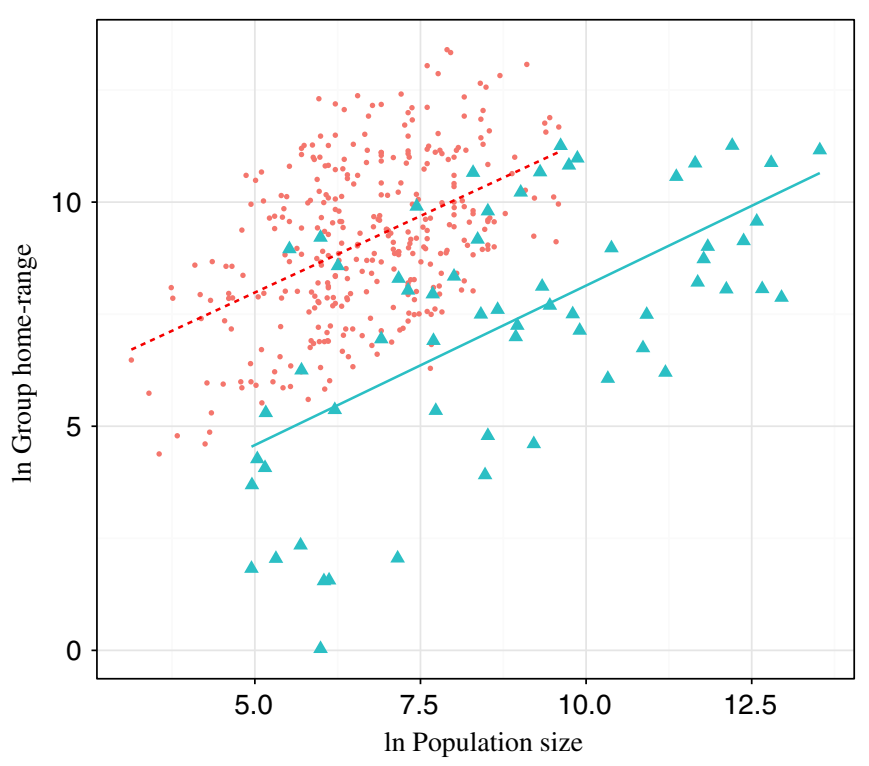

Figure 2 Population-territory size scaling. Dots=hunter-gatherer societies; triangles=agricultural societies. The dashed line is an OLS regression line for hunter-gatherers; the solid line is the same for agriculturalists. Reproduced from Freeman (2016).

A common way to model the possibility that the relationship between population and energy consumption might be sub-linear is with a power function:

$$
E=m_{2} P^{\beta_{2}}
$$

where $E$ is the total energy consumed by a population; $m_{2}$ is a scaling constant; and $\beta_{2}$ is the scaling exponent. Here, population is constrained to positive integers, and we assume that $\beta_{2}>0$ (see Figure $1 \mathrm{~b}$ ). Where $\beta_{2}$ is equal to one, population scales linearly with energy consumption; $0<\beta_{2}<1$ the scaling is sub-linear; and $\beta_{2}>0$ the scaling is super-linear.

Given Equations 2 and 3, we can combine the constants of $m_{1}$ and $m_{2}$ and set $m_{1} \times m_{2}=M_{0}$, and rewrite Equation 1 as

$$
E=M P=M_{0} e^{\beta_{1} C} P^{\beta_{2}}
$$

In sum, Equation 4 states that the total energy consumed by a population is biological metabolism times economic complexity, which defines a cultural metabolism per person to live and reproduce, times the total number of people in a population.

\section{METHODS AND DATA}

Equation 4 allows us to investigate the scaling relationship between population size and energy consumption, holding economic complexity constant. We can evaluate whether the scaling of population and energy consumption is sub-linear in contemporary contexts by, first, taking the log of the right and left hand sides of Equation 4 to obtain a linear model:

$$
\ln E=\ln M_{0}+\beta_{1} C+\beta_{2} \ln P+\epsilon
$$


where $\varepsilon$ is the variability in the log of energy consumption not explained by population and complexity. We use the log transformations to make Equation 4 linear because this allows us to use well established techniques for estimating $\beta_{2}$ using a linear regression model.

To estimate the coefficients and intercept in Equation 5, we use robust linear regression. Robust linear regression refers to a set of regression models that are more robust to the effects of outliers than the ordinary least-squares method. Here, we specifically use maximum likelihood type estimation with the Huber weight function, by running the MASS package and "rlm()" function in R. This method of regression is robust to outliers in the response variable (ln $E$ below). We calculate $95 \%$ confidence interval estimates for $M_{0}, \beta_{1}$, and $\beta_{2}$ by bootstrapping with replacement 2000 times.

To evaluate the relationship between population and energy consumption, we use four data sets that document the relationship in contemporary societies. In the first, we use International Energy Agency estimates of total energy consumption (IEA 2016) in 146 countries in 2013 and population estimates for each country from the World Bank in 2013 (TWB 2016). The energy consumption data are self reported by each of the countries in the data set. The data, thus, come from countries with mainly subsistence economies to countries with post-industrial knowledge economies (e.g., Tanzania versus Japan), and the data vary in accuracy, which is a source of measurement error. We have made an attempt to control for variability in the consumption of energy driven by big differences in economic complexity by collecting data from Hausmann et al. (2014) on economic complexity. The data are available for 117 countries that also have population and energy consumption data. Hausmann et al. (2014) measure economic complexity using an index that captures the diversity and ubiquity of products in an economy to create an export network, and they argue that these networks reflect the capabilities and degree of capability integration in an economy. The larger the number of products and the more unique, the more organizationally complex the economy. This, of course, assumes that the index of economic complexity is positively and linearly related to the true level of complexity in an economy. There are other ways one might estimate complexity, but this is a point of departure that might be useful for archaeologists since we can observe the diversity and ubiquity of material products in the record (though understanding export and import dynamics is highly constrained). Using these data, we can examine the scaling relationship between population and energy consumption holding economic complexity constant.

In the second data set, we use estimates of total energy consumption in U.S. states in 2014 obtained from the United States Energy Information Administration (EIA) (EIA 2016) and estimates of population in 2014 obtained from the U.S. Census (Census 2016). These data are standardized estimates of energy consumption collected by energy professionals at the EIA. We treat economic complexity among U.S. states as a constant because the variation in complexity is less than the global sample. This means that all U.S. states are similar in economic complexity to say France, but no state approaches the lower 1/2 of the economic complexity distribution observed at a global scale. In the global and U.S. data sets we combine all forms of energy consumption (e.g., wind, solar, nuclear, coal) and sectors (e.g., transportation, industrial, residential). We are not concerned that many of these energy sources would not result the production of datable materials, but, rather, with describing the general scaling relationship between population and energy consumption.

In the third data set, we look at the relationship between the number of families in villages and wood-fuel consumption in Bangladesh (Miah et al. 2009:Table 2). This data set has a sample 
size well below 30 and, thus, standard errors are likely inflated. Yet, the data set is instructive because we can observe energy consumption at a much smaller scale and level of analysis than in the first two data sets. These data were collected among subsistence farmers as part of an ethnographic study on fuel consumption and deforestation. Again, economic complexity varies little from village-to-village in this data set, so we treat complexity as a constant.

Finally, in the fourth data set we observe the relationship between the population size of Kalahari Bushman camps and the number of hearths in each camp. We assume that the number of hearths is a proxy for the amount of fuel-wood and other organics consumed by the population of each camp to cook, stay warm, etc. Thus, we treat the number of hearths in a camp as an estimate of energy consumption among these hunter-gatherers. All data come from Yellen's (1977) ethnoarchaeological study of Kalahari Bushman. We tabulated the data from Yellen's camp descriptions. We estimated the total number of hearths in a camp by adding the formal hearths described for each camp with informal hearths. We estimated the number of informal hearths using Yellen's feature list for each camp site and by tabulating the number of small one-time or special-purpose roasting pits, scatters or mounds of charcoal and ash that might be fire hearths. We avoided any that he specifically listed as hearth clean-outs, but it is possible that some informal hearths are clean-outs. This gave us a data set of 15 camps. As above, the caveat about small sample size applies, and economic complexity varies little from camp-to-camp, so we treat complexity as a constant.

We would like to emphasize that we are using data from three different scales (global, national, and local), and these data come from a wide variety of economies. One might worry that differences in economic complexity might change the population-energy consumption relationship; this is why we have taken the time and care to build data sets at very different scales and from a range of economic contexts. Convergent results might suggest that population has a widespread effect on energy consumption that transcends types of economies.

\section{RESULTS}

In sum, our results indicate that, at a global scale of analysis, population size and economic complexity both have effects on the total energy consumed by a population. Further, at a global scale, the scaling relationship between population and energy consumption, holding economic complexity equal, is sub-linear. The sub-linear scaling of population and energy consumption is replicated at finer scales of analysis where economic complexity varies much less than at a global scale. The scaling of population and energy consumption is sub-linear among U.S. states, Bangladesh villages and Kalahari Bushman camps. Remarkably, the scaling coefficients identified in our analysis are similar to the population-fuel-wood consumption (energy consumption) coefficient of 0.79 (s.e. =0.04), controlling for forest cover and GDP, found by Knight and Rosa (2012:Table 2) in their study of wood consumption in 87 developing economies. This means that a sub-linear scaling of population and energy consumption, holding other factors equal, occurs across five different data sets collected at different scales of analysis and with very different levels of technological variation.

Table 1 illustrates that the scaling relationship between population and energy consumption is sub-linear, even after controlling for economic complexity, at the global scale. As expected, population has a positive effect on energy consumption, with a sub-linear coefficient of $\beta_{2}=0.85$. Also, as expected, economic complexity has a positive effect on energy consumption. The more complex a country's economy, the more energy the population of a country consumes. Finally, although both population size and economic complexity have 
Table 1 Parameter estimates, standard errors and confidence intervals for the regression analysis of each dataset: (a) global country level data, (b) U.S. state data, (c) Bangladesh villages, and (d) Kalahari camps. Note that the estimated $y$-intercepts $\left(\ln M_{0}\right)$ in regressions b-d should be interpreted as the sum of the log-linear model's $\ln M_{0}$ and $\beta_{1} C$ term because these respective parameters are not statistically identifiable when we assume that the variable $C$ is constant.

\begin{tabular}{lccc}
\hline Parameter & Coefficient & Std. error & $95 \%$ C.I. \\
\hline (a) Global regression & & & \\
$\ln M_{0}$ & -11.33 & 0.80 & $-12.84,-9.67$ \\
$\beta_{1}$ & 0.48 & 0.05 & $0.37,0.59$ \\
$\beta_{2}$ & 0.85 & 0.04 & $0.76,0.94$ \\
(b) U.S. state regression & & & $-7.85,-4.02$ \\
$\ln M_{0}$ & -5.91 & 0.99 & $0.73,0.98$ \\
$\beta_{2}$ & 0.86 & 0.06 & $-8.61,2.15$ \\
(c) Bangladesh regression & & & $0.55,2.00$ \\
$\ln M_{0}$ & 1.66 & 1.20 & $-3.16,1.91$ \\
$\beta_{2}$ & 0.89 & 0.49 & $0.02,1.78$ \\
(d) Kalahari regression & & & 1.33 \\
$\ln M_{0}$ & -0.64 & 0.46 & \\
$\beta_{2}$ & 0.89 & &
\end{tabular}

(a)

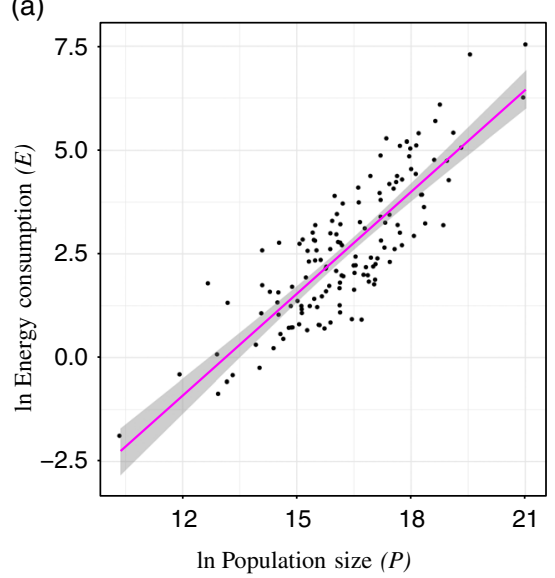

(b)

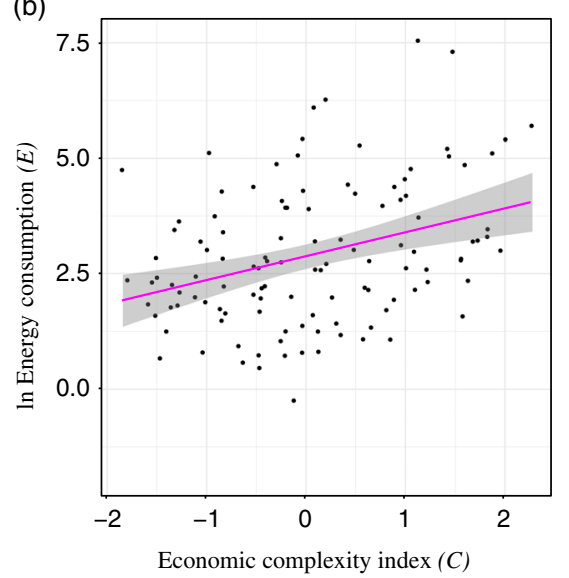

Figure 3 (a) The relationship between population and total energy consumption among world countries. (b) The relationship between the economic complexity index and total energy consumption among world countries.

effects on energy consumption consistent with theory; population size explains more of the variability in energy consumption than economic complexity. This is intuitively illustrated by Figure 3 . Note that the points are a much tighter fit around the best fit line in Figure $3 a$ versus $3 b$.

Table 1 also illustrates that the scaling of population size and energy consumption is sub-linear at finer scales of analysis where economic complexity is much less variable among U.S. states, Bangladesh villages and Bushman camps. Among U.S. states, the scaling of population and energy consumption is sub-linear at $\beta_{2}=0.86$. Among Bangladesh villages the scaling of 
population and fuel-wood consumption is again sub-linear, at $\beta_{2}=0.89$. However, due to the very small sample size, the standard error of $\beta_{2}$ in this case is quite large, and $\beta_{2}=1$, as well as $\beta_{2}>1$ are within the $95 \%$ confidence interval for the scaling exponent. Among Bushman camps the scaling of population and number of hearths is sub-linear, at $\beta_{2}=0.89$. As with the Bangladesh villages, the sample size and standard error caveats apply.

\section{DISCUSSION}

So far, we have proposed a model that scales population against the production of datable materials in social-ecological systems, and we have parametrized the model using modern data on population size and energy consumption. We assume that a given unit of increase in the energy consumed by a prehistoric population results in a proportionate increase in the accrual of materials that archaeologists ultimately date. Contemporary data sets demonstrate a sub-linear scaling relationship between population size and total energy consumption, and do so at three different levels of analysis and in five different data sets (Table 1). Further, at a global scale, where variation in economic complexity is widest, economic complexity has a positive effect on the consumption of energy (Table 1, Figure 3).

The model proposed by Equation 4 and the results of our analysis, which are consistent with Equation 4, suggest two points relevant to the study of dates as data. (1) ${ }^{14} \mathrm{C}$ date frequencies arrayed in a time-series, based on large samples of dates, as currently constructed, probably misestimate rates of population growth. This is because ${ }^{14} \mathrm{C}$ dates, all else equal, are one estimate of the energy consumed in prehistoric social-ecological systems, and the scaling relationship between population and energy consumption is often sub-linear in human populations. (2) Equation 4 provides a framework to make predictions about how the ${ }^{14} \mathrm{C}$ record should covary with other classes of archaeological material culture and further evaluate the importance of changes in energy consumption in prehistoric social change.

\section{Estimating Population Size and Growth}

The sub-linear scaling of population size with energy consumption documented above suggests that current approaches to interpreting ${ }^{14} \mathrm{C}$ date frequencies systematically misestimate population size over a given interval of time and growth rates. This is not a concern if we don't care about absolute population sizes and growth rates. For example, some researchers pool together dates that are associated with the same context/site (e.g., Downey et al. 2014; Shennan et al. 2013; Timpson et al. 2014). This is done to control for sampling bias by archaeaologists, and shifts the frequency of ${ }^{14} \mathrm{C}$ dates from an estimate of the number of individuals to an estimate of the number of sites or number of phases of occupation. Counting sites is a classic method that archaeologists use to estimate changes in population. This method is probably pretty good for understanding relative changes in population over time. Indeed, Downey et al. (2014) have conducted work that parallels our own in certain respects. They recognize that the population of settlements varies with subsistence technology, and that there is a scaling between settlement population and settlement area. Thus, they adjust their estimates of population from site counts by these factors to obtain more informed estimates of prehistoric population sizes and rates of change. This is valuable work relevant to making inferences about prehistoric population parameters.

However, the method of pooling together dates that are associated with the same context/site degrades the information contained within the ${ }^{14} \mathrm{C}$ record. The model we develop is about the factors that create variation in the ${ }^{14} \mathrm{C}$ record itself. With such a model, one can adjust the ${ }^{14} \mathrm{C}$ 
record to make more informed estimates of population, but the underlying model is about the factors (internal to prehistoric systems) that create this variation in the first place. We may want to use the ${ }^{14} \mathrm{C}$ record for more than just estimating relative demographic parameters. We may want to estimate changes in the production of waste more generally, or we may want to know about absolute values of population size and change. In this case, we need to think about how to adjust frequencies of ${ }^{14} \mathrm{C}$ dates to account for the non-linear relationship between population and energy consumption, without simply using them to count sites, which has the benefit of controlling for sampling intensity but also has the cost of lost information.

Given our model and results, we propose, as a thought experiment, a method for rescaling ${ }^{14} \mathrm{C}$ datasets to estimate relative population sizes that accounts for a sub-linear relationship between population size and the evidence for energy consumption. To conduct this thought experiment we hold economic complexity constant because this factor appears to determine less variation in energy consumption than population size. It is the evidence of energy consumption events, not population size, that archaeologists analyze, and the available data suggest a sub-linear relationship between population size and the consumption of energy. Starting with Equation 4, since $M_{O}$ is a constant, we can let $M_{0}=1$, hold $C=1$, and solve for $P$ at a given time $t$ by raising each side of the equation to a power of $1 / \beta$ :

$$
P_{t}=E_{t}^{\left(\frac{1}{\beta}\right)}
$$

Taking our contemporary data as a starting point, we could rescale the frequency of dates in any given time period by a scaling factor between $1.12-1.26$, (i.e., $1 / \beta$ ) for the range of values derived above from the modern data sets (Knight and Rosa 2012:Table 2; Table 1 in this study). It is important to note that by doing this we assume that the sub-linear scaling of population and energy consumption (and, inversely, the super-linear scaling of energy consumption and population) is an invariant law of human social-ecological dynamics. This is a strong assumption, and one we make with caution. For now, however, a hypothetical scaling exponent of 1.15 recognizes the sub-linear relationship between population and the production of datable material. Doing this does two things: It raises estimates of population size in any given time interval, and it increases estimates of population growth.

In this case, the rate of change in the scaled summed probability distribution (SPD) is $15 \%$ faster, and, thus, the estimated growth rate $15 \%$ faster than an un-scaled SPD of ${ }^{14} \mathrm{C}$ dates (the dashed curve increases faster than the solid curve in Figure 4). In terms of real numbers, Zahid et al. (2016:934) estimate the annual population growth rate of SW Wyoming and Colorado, using an SPD uncorrected for taphonomic loss, as $0.053 \%$. Our results suggest that this underestimates population growth from between $12 \%$ to $26 \%$, which yields growth rates from $0.05936 \%$ to $0.0667 \%$. Simply put, an unscaled SPD underestimates population size, and, because the relationship between energy consumption and population may be sub-linear, an unscaled SPD will also underestimate rates of population growth.

For context, Zahid et al. (2016:932) correct their raw growth rate of $0.053 \%$ for taphonomic loss to obtain a growth rate of $0.041 \%$ during the period from 13,000 to $6000 \mathrm{cal} \mathrm{BP}$. Thus, in absolute terms, the energy scaling adjustment is comparable to the taphonomic adjustment. In fact, deep in time both adjustments cancel each other out. However, as one moves closer to the present, taphonomic loss is less important and an adjustment for population-energy scaling would have more of an effect. In relative terms, at low population sizes, neither adjustment probably matters all that much.

However, the energy scaling adjustment applies through a whole time-series at a constant rate; and a change of $15 \%$ in growth rate may be more meaningful for larger populations, and over 


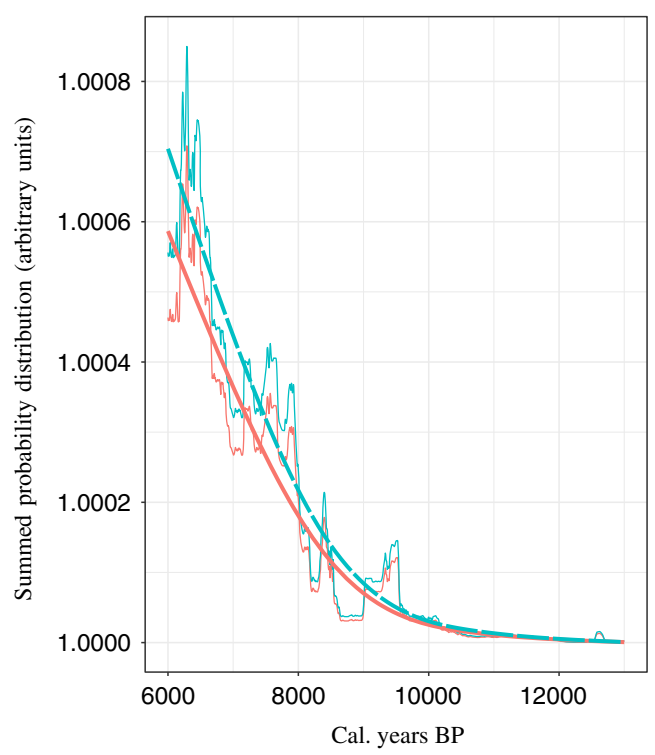

Figure 4 Raw and transformed SPDs. Dashed blue curve is the best fit for the transformed SPD; the solid red curve is the best fit for the raw SPD. (See online version for colors.)

time periods in which populations were growing at faster rates. For example, if a population of 1000 experienced a period of exponential growth at $1 \%$ versus $1.15 \%$ over 200 years, the population growing at $1 \%$ would have a population of approximately 7316 people and the population growing at $1.15 \%$ a population of approximately 9844 people (about a $35 \%$ difference). Keep in mind also that the energy scaling adjustment factor also changes absolute population sizes and, holding space constant, population density. Theory suggests that critical population density thresholds fundamentally change the selective pressures put on individuals (Winterhalder et al. 1988; Binford 1999; Freeman and Anderies 2012; Freeman et al. 2015). If we are systemically underestimating population densities, we may be missing evidence that such thresholds were approached and crossed.

In sum, if the goal of a project is to reconstruct absolute population densities or growth rates, then an informed researcher might transform their ${ }^{14} \mathrm{C}$ date curve to account for the non-linear scaling of energy consumption and population size, as documented here. To be clear, we are not suggesting that all SPDs need to be adjusted to account for the sub-linear scaling of population and energy consumption. Rather, if absolute growth rates are important, then we need to build frames of reference useful for estimating absolute growth rates from SPD data. Conceptualizing ${ }^{14} \mathrm{C}$ date timeseries as estimates of energy consumption, thus, does not preclude using SPDs to estimate demographic parameters, but rather, gives us a more informed way to do so. This is just one advantage of developing a model framework, from the factors that should drive energy consumption in human societies, for predicting variation ${ }^{14} \mathrm{C}$ date frequencies. In fact, our work may be useful to incorporate into dynamic growth models that take into account changes in competition and exogenous factors, like fluctuations in climate (Brown 2017).

\section{Predicting Covariates in the Archaeological Record}

We know from previous research that archaeologists must control for the effects of calibration, over sampling of single features or sites (sampling intensity), under certain circumstances, 
taphonomic processes, and even must be cognizant of the assumptions of different growth models before inferring population parameters from large ${ }^{14} \mathrm{C}$ time-series (Surovell et al. 2009; Williams 2012; Contreras and Meadows 2014; Brown 2015, 2017). Proper ${ }^{14} \mathrm{C}$ hygiene limits over-sampling induced bias, and recent research has shown that large sample sizes (1000+ assays) are more robust to the effects of preservation bias (Williams 2012). Much work has gone into these issues. Intuition probably tells most archaeologists that these issues are more important than the social dynamics of prehistoric populations that created the ${ }^{14} \mathrm{C}$ record through energy consumption events. This, however, is an empirical question, and our approach does not reject the importance of sampling bias and taphonomic processes. Our approach simply puts processes external and internal to prehistoric systems on a more equal empirical footing so that we can begin to tease apart the most important factors.

As a final discussion point, the theoretical framework developed above provides a starting point to observe the relationships between ${ }^{14} \mathrm{C}$ date frequencies and other classes of phenomena related to energy consumption in the archaeological record. We do not argue that ${ }^{14} \mathrm{C}$ date frequencies are a sufficient measure of energy consumption. Rather, the ${ }^{14} \mathrm{C}$ record is one estimate of energy consumption. We can correlate the ${ }^{14} \mathrm{C}$ record with other estimates of energy consumption using other classes of material culture to evaluate this idea further. For example, if the ${ }^{14} \mathrm{C}$ curve is an estimate of total energy consumption, we would expect to see spikes in the ${ }^{14} \mathrm{C}$ curve correlate with the use of more energy dense biomass, like grass seeds and nuts, as opposed to less energy dense resources on the landscape. Hence macrobotanical evidence of seed use should spike or evidence of agricultural intensification should spike as the ${ }^{14} \mathrm{C}$ record spikes, depending on the region and economic context. The model we have proposed is not an end product, but is a beginning theory, justified by basic relationships between population and energy consumption in modern contexts, and these modern relationships suggest that the model may prove useful in archaeological contexts as well.

\section{CONCLUSION}

The purpose of this paper has been to critically discuss how to observe prehistoric social and demographic processes from large samples of ${ }^{14} \mathrm{C}$ dates. While much research has been conducted to model and investigate non-cultural biasing agents, less attention has been given to modeling how prehistoric culture process may affect the accumulation of datable materials in prehistoric social-ecological systems and, thus, the amount of material available for archaeologists to date. Consequently, we suggest that understanding the energy consumption dynamics of human societies represents both a critical and logical step to make predictions that explain variation in ${ }^{14} \mathrm{C}$ date time-series.

We propose that large, regional-scale samples of ${ }^{14} \mathrm{C}$ dates estimate changes in the consumption of energy in prehistoric populations rather than population per se. It is important to note that this is just one estimate. If this approach has validity, then future research will show clear positive covariation between frequencies of ${ }^{14} \mathrm{C}$ dates and other material estimates of energy consumption, like the frequency of ground stone or the size of middens. Our results suggest that energy consumption is a sub-linear function of population size and is positively related to economic complexity, at a global scale. Given these relationships, if one is interested in using ${ }^{14} \mathrm{C}$ data to estimate population growth rates, it may be productive to adjust a resultant timeseries to estimate population sizes and changes in population over time. This adjustment should not be viewed as a "correction" of a ${ }^{14} \mathrm{C}$ curve. Correction implies that a given curve is wrong. Rather, the adjustment represents an informed judgment that should be made if one's research goal is to estimate absolute population growth rates, and if future research supports the 
hypothesis that the scaling of population size and energy consumption is sub-linear. We have presented this correction example as a thought experiment; as a challenge to make us think about what kind of correction we might need and when.

Large samples of ${ }^{14} \mathrm{C}$ dates are a potentially informative way to measure prehistoric culture process. More work is needed, however. We suggest three lines of research that may complement the already vigorous research into how best to make inferences from ${ }^{14} \mathrm{C}$ time-series.

- Collect more data on contemporary or ethnographically recorded economies to empirically investigate the scaling relationship between population and energy consumption. If the scaling of population and energy consumption is an invariant law driven by basic metabolic processes (Brown et al. 2004; Hamilton et al. 2007), this would be incredibly convenient for archaeologists interested in making inferences from large ${ }^{14} \mathrm{C}$ data sets. However, the scaling may vary with technology or social institutions over time and space (DeLong and Burger 2015; Freeman and Anderies 2015), which would mean that different parts of the ${ }^{14} \mathrm{C}$ date time-series would need to be rescaled, from the perspective of estimating population size and growth rates, by different scaling factors over different segments of time.

- Evaluate the scaling of ${ }^{14} \mathrm{C}$ time-series and time series of other classes of archaeological material culture. For instance, the ${ }^{14} \mathrm{C}$ record should scale sub-linearly with alternative estimates of population, such as structure counts.

- Study further the effects of economic and political complexity on energy consumption. Fluctuations in ${ }^{14} \mathrm{C}$ date time-series curves, holding all else equal, also result from changes is social and economic organization (Crombé and Robinson 2014). Holding population constant, changes in economic organization, as well as complexity, should affect how much energy an average individual consumes.

To end, we would like to emphasize again that our contribution is theoretical. We have proposed a quantitative model to describe the relationship between the production of ${ }^{14} \mathrm{C}$ dates and human population. The practical relevance is twofold. First, the model we have specified allows us to predict how ${ }^{14} \mathrm{C}$ dates should covary with other classes of archaeological material culture. Second, and the nominal focus of our paper, the model allows us to make better judgments about how to infer prehistoric population parameters from large samples of ${ }^{14} \mathrm{C}$ dates. Our approach is not a finished product, but it is a step toward a more mature, deductive approach to learning about social and demographic processes from large samples of ${ }^{14} \mathrm{C}$ dates.

\section{ACKNOWLEDGMENTS}

This paper has been greatly improved by the contributions of two anonymous reviewers. We thank them. This paper is a product of the "Coevolution of human population, climate and food systems" workshop funded by Past Global Changes (PAGES) at USU in 2016. We would like to thank PAGES and the workshop participants for support and stimulating conversations that motivated this paper. The first author would like to acknowledge the support of the National Science Foundation (IBSS-L: Award \# 1520308). We would also like to thank Ray Mauldin for valuable comments on an earlier version of this manuscript. All mistakes are our own.

\section{SUPPLEMENTARY MATERIAL}

To view supplementary material for this article, please visit https://doi.org/10.1017/RDC. 2017.124 


\section{REFERENCES}

Attenbrow V, Hiscock P. 2015. Dates and demography: are radiometric dates a robust proxy for long-term prehistoric demographic change? Archaeology in Oceania 50:30-6.

Binford LR. 1999. Time as a clue to cause? Proceedings of the British Academy 101:1-35.

Brown JH, Gillooly JF, Allen AP, Savage VM, West GB. 2004. Toward a metabolic theory of ecology. Ecology 85:1771-89.

Brown WA. 2015. Through a filter, darkly: population size estimation, systematic error, and random error in radiocarbon-supported demographic temporal frequency analysis. Journal of Archaeological Science 53:133-47.

Brown WA. 2017. The past and future of growth rate estimation in demographic temporal frequency analysis: biodemographic interpretability and the ascendance of dynamic growth models. Journal of Archaeological Science 80:96-108.

Census. 2016. State population totals datasets: 2010 2016 American Community Survey. https://www. census.gov/data/datasets/2016/demo/popest/statetotal.html.

Contreras DA, Meadows J. 2014. Summed radiocarbon calibrations as a population proxy: a critical evaluation using a realistic simulation approach. Journal of Archaeological Science 52:591-608.

Crema ER, Habu J, Kobayashi K, Madella M. 2016. Summed probability distribution of ${ }^{14} \mathrm{C}$ dates suggests regional divergences in the population dynamics of the Jomon Period in Eastern Japan. PLOS ONE 11:e0154809.

Crombé P, Robinson E. $2014 .{ }^{14} \mathrm{C}$ dates as demographic proxies in Neolithisation models of northwestern Europe: a critical assessment using Belgium and northeast France as a case-study. Journal of Archaeological Science 52:558-66.

DeLong JP, Burger O. 2015. Socio-economic instability and the scaling of energy use with population size. PLoS ONE 10:e130547.

Downey SS, Bocaege E, Kerig T, Edinborough K, Shennan S. 2014. The Neolithic Demographic Transition in Europe: correlation with juvenility index supports interpretation of the summed calibrated radiocarbon date probability distribution (SCDPD) as a valid demographic proxy. PloS One 9:e105730.

EIA. 2016. State comparisons, The U.S. Energy Information Administration. https://www.eia. gov/state/compare/.

Freeman J. 2016. The socioecology of territory size and a "work-around" hypothesis for the adoption of farming. PLoS ONE 11:e0158743.

Freeman J, Anderies JM. 2012. Intensification, tipping points and social change in a coupled forager-natural resource system. Human Nature 23:419-46.
Freeman J, Anderies JM. 2015. The socioecology of hunter-gatherer territory size. Journal of Anthropological Archaeology 39:110-23.

Freeman J, Peeples M, Anderies JM. 2015. Toward a non-linear theory of the transition from foraging to farming. Journal of Anthropological Archaeology 40:109-22.

Hamilton MJ, Burger O, DeLong JP, Walker RS, Moses ME, Brown JH. 2009. Population stability, cooperation, and the invasibility of the human species. Proceedings of the National Academy of Sciences of the United States of America 106(12): 255-60.

Hamilton MJ, Milne BT, Walker RS, Brown JH. 2007. Nonlinear scaling of space use in human hunter-gatherers. Proceedings of the National Academy of Sciences of the United States of America 104:4765-9.

Hausmann R, Hidalgo CA, Bustos S, Coscia M, Simoes A, Yildirim MA. 2014. The Atlas of Economic Complexity: Mapping Paths to Prosperity. MIT Press.

Hidalgo CA, Hausmann R. 2009. The building blocks of economic complexity. Proceedings of the National Academy of Sciences 106(10):570-5.

IEA. 2016. World energy balance data, International Energy Agency. https://www.iea.org/Sankey/.

Jetz W, Carbone C, Fulford J, Brown J. 2004. The scaling of animal space use. Science 306:266-8.

Kelly RL, Surovell TA, Shuman BN, Smith GM. 2013. A continuous climatic impact on Holocene human population in the Rocky Mountains. Proceedings of the National Academy of Sciences 110:443-7.

Knight KW, Rosa EA. 2012. Household dynamics and fuelwood consumption in developing countries: a cross-national analysis. Population and Environment 33:365-78.

Lindstedt SL, Miller BJ, Buskirk SW. 1986. Home range, time, and body size in mammals. Ecology 67:413-8.

McNab BK. 1963. Bioenergetics and determination of home range size. American Naturalist 97:133-40.

Miah MD, Al Rashid H, Shin MY. 2009. Wood fuel use in the traditional cooking stoves in the rural floodplain areas of Bangladesh: a socioenvironmental perspective. Biomass and Bioenergy 33:70-8.

Milton K, May ML. 1976. Body-weight, diet and home range area in primates. Nature 259:459-62.

Peros MC, Munoz SE, Gajewski K, Viau AE. 2010. Prehistoric demography of North America inferred from radiocarbon data. Journal of Archaeological Science 37:656-64.

Pettitt P, Davies W, Gamble C, Richards M. 2003. Palaeolithic radiocarbon chronology: quantifying our confidence beyond two half-lives. Journal of Archaeological Science 30:1685-93. 
Rick JW. 1987. Dates as data: an examination of the Peruvian Preceramic radiocarbon record. American Antiquity 52:55-73.

Shennan S. 2008. Population processes and their consequences in early Neolithic central Europe. In: The Neolithic Demographic Transition and its Consequences. Springer. p. 315-29.

Shennan S. 2013. Demographic continuities and discontinuities in Neolithic Europe: evidence, methods and implications. Journal of Archaeological Method and Theory 20:300-11.

Shennan S, Downey SS, Timpson A, Edinborough K, Colledge S, Kerig T, Manning K, Thomas MG. 2013. Regional population collapse followed initial agriculture booms in mid-Holocene Europe. Nature Communications 4.

Surovell TA, Byrd Finley J, Smith GM, Brantingham PJ, Kelly R. 2009. Correcting temporal frequency distributions for taphonomic bias. Journal of Archaeological Science 36:1715-24.

Timpson A, Colledge S, Crema E, Edinborough K, Kerig T, Manning K, Thomas MG, Shennan S. 2014. Reconstructing regional population fluctuations in the European Neolithic using radiocarbon dates: a new case-study using an improved method. Journal of Archaeological Science 52:549-57.

TWB. 2016. World development indicators, The World Bank. http://data.worldbank.org/datacatalog/world-development-indicators.
Wang C, Lu H, Zhang J, Gu Z, He K. 2014. Prehistoric demographic fluctuations in China inferred from radiocarbon data and their linkage with climate change over the past 50,000 years. Quaternary Science Reviews 98: 45-59.

Williams AN. 2012. The use of summed radiocarbon probability distributions in archaeology: a review of methods. Journal of Archaeological Science 39:578-89.

Williams AN. 2013. A new population curve for prehistoric Australia. Proceedings of the Royal Society B: Biological Sciences 280:2013 0486.

Winterhalder B, Baillargeon W, Cappelletto F, Daniel I, Prescott C. 1988. The population ecology of hunter-gatherers and their prey. Journal of Anthropological Archaeology 7:289-328.

Yellen JE. 1977. Archaeological Approaches to the Present: Models for Reconstructing the Past. Volume 1. Academic Press.

York R, Rosa EA, Dietz T. 2003. STIRPAT, IPAT and ImPACT: analytic tools for unpacking the driving forces of environmental impacts. Ecological Economics 46:351-65.

Zahid HJ, Robinson E, Kelly RL. 2016. Agriculture, population growth, and statistical analysis of the radiocarbon record. Proceedings of the National Academy of Sciences 113:931-5. 